\title{
Integration with respect to a trace
}

By

Michael Leinert

J. Dixmier [4] and I. Segal [14] started noncommutative integration. If one is interested in the abstract $L^{p}$ spaces (obtained by completion), one finds many of the known facts already in [4]. Segal [14] defined concrete $L^{p}$ spaces, the elements thereof being certain unbounded operators. His work was continued by Kunze [8] and Stinespring [15]. Nelson in his elegant paper [12] considered the abstract $L^{p}$ spaces and then injected them into the unbounded operators. We prefer to work with the unbounded operators themselves, thus following Segal. The upper integral which we use makes things fairly easy. After the properties of the upper integral have been established, almost all the rest works by easy standard proofs or even commutative proofs (i.e. proofs which one uses in ordinary measure theory) plus some facts from complex interpolation. This note grew out of an attempt to see whether the approach of [9] works in the noncommutative case. The reader who is not interested in details of proofs may prefer to read the outline given in [10]. The general case of integration with respect to a weight is treated in [11].

In Section 1 we consider the space $M$ of all (strongly) measurable operators. We use Segal's definition of measurability with "algebraically finite" replaced by "of finite trace". $M$ is identical with the image of $\tilde{\mathfrak{U}}$ in [12], Theorem 4. In Section 2 we define the upper integral $\bar{\varphi}$, show that it is a countably additive integral on $M^{+}$and prove a Beppo Levi Theorem. In Section 3 we define $L^{p}$. The classical arguments used to show that $L^{1}$ is a normed linear space work in our context, too. As a consequence of the Beppo Levi Theorem we have completeness of $L^{1}$ and the Monotone Convergence Theorem. We continue with Egoroff's Theorem, Lebesgue's Dominated Convergence Theorem, and the duality $\left(L^{1}\right)^{*}=\mathfrak{A}$. This duality is now a Radon-Nikodym result (a benefit of the fact that we work in the concrete situation of unbounded operators). In Section 4 we show that the set $L^{p}$ coincides with the complex interpolation space $\left(\mathscr{2 I}, L^{1}\right)_{1 / p}$. As a consequence, $L^{p}$ is a Banach space and $\left(L^{p}\right)^{*}=L^{q}\left(\right.$ where $\left.\frac{1}{p}+\frac{1}{q}=1\right)$. Section 5 contains two alternative formulations of the upper integral leading to a stronger variant of our approach and some remarks on convergence of measurable operators in measure, a.e., and almost uniformly.

I wish to thank Michael Cowling for discussions and for introducing me to interpolation spaces. 
Let $H$ be a Hilbert space, $T$ a linear operator on $H$, bounded or not. By $D(T), N(T), R(T)$ we denote the domain of definition, the null space, and the range of $T$, respectively. If $T$ is closable, $\bar{T}$ denotes its closure. Let $\mathfrak{I}$ be a von Neumann algebra on $H, \mathfrak{I}^{+}$its positive part, $\mathfrak{I I}^{\prime}$ its commutant. A linear operator $T$ is called affiliated with $\mathfrak{A}$ (in symbols: $T \sim \mathfrak{A}$ ), if $T U=U T$ for all unitary $U \in \mathfrak{A}^{\prime}$. This equality includes domains, of course, so in particular it is required that $U D(T)=D(T)$ for all unitary $U \in \mathfrak{A}^{\prime}$, or equivalently $U D(T) \subset D(T)$ for all unitary $U \in \mathfrak{A}^{\prime}$. Let $\varphi=\mathfrak{A}^{+} \rightarrow[0, \infty]$ be a trace, i.e. a functional satisfying

(i) $\varphi(\lambda A)=\lambda \varphi(A)$ for $\lambda \geqq 0, A \in \mathfrak{A}^{+}$(with $\left.0 \cdot \infty \stackrel{\text { def }}{=} 0\right)$

(ii) $\varphi(A+B)=\varphi(A)+\varphi(B)$ for $A, B \in \mathfrak{U}^{+}$

(iii) $\varphi\left(A^{*} A\right)=\varphi\left(A A^{*}\right)$ for $A \in \mathfrak{U}$.

For $B \in \mathfrak{U}^{+}$and a partial isometry $u \in \mathfrak{I}$ one has by (iii)

$$
\varphi\left(u B u^{*}\right)=\varphi\left(B^{1 / 2} u^{*} u B^{1 / 2}\right) \leqq \varphi(B)
$$

since $B^{1 / 2} u^{*} u B^{1 / 2} \leqq\left\|u^{*} u\right\| B \leqq B$.

We suppose the trace $\varphi$ to be faithful, i.e. $\varphi(A)=0$ implies $A=0$, semifinite, i.e. $\varphi(A)=\sup \left\{\varphi(B) \mid B \in \mathfrak{A}^{+}, B \leqq A, \varphi(B)<\infty\right\}$, and normal, i.e. for any increasing net $\left\{\mathbb{T}_{\mu}\right\}$ in $\mathfrak{U}^{+}$with $T_{\mu} \nearrow T \in \mathfrak{U}^{+}$in the weak operator topology, one has $\varphi\left(T_{\mu}\right) \nearrow \varphi(T)$. The normality condition may be restated as follows:

For $T \in \mathfrak{A}^{+}$and any increasing net $\left\{T_{\mu}\right\}$ in $\mathfrak{U}^{+}$with $\lim \left(T_{\mu} x \mid x\right) \geqq(T x \mid x)$ for all $x \in H$, one has $\lim \varphi\left(T_{\mu}\right) \geqq \varphi(T)$ (the limits being possibly infinite).

This condition clearly implies normality. It is equivalent to normality, because for every normal trace $\varphi$ there is a family of vectors $\left\{x_{i}\right\}_{i \in I}$ in $H$ such that $\varphi(T)=\sum_{i \in I}\left(T x_{i} \mid x_{i}\right)$ (see [5], p. 85).

The word "projection" always means "orthogonal projection". For projections $p_{n} \in \mathfrak{A}$ with $\sum p_{n}=1$ weakly, one has $\sum A^{1 / 2} p_{n} A^{1 / 2}=A$ weakly, so by normality and (iii) we obtain

$$
\varphi(A)=\sum \varphi\left(A^{1 / 2} p_{n} A^{1 / 2}\right)=\sum \varphi\left(p_{n} A p_{n}\right) .
$$

If $S$ is a linear subspace of $H, p_{S}$ denotes the projection onto the closure $\bar{S}$ of $S$. If $r, s$ are projections, $r \cap s$ and $r \cup s$ denote $p_{r H \cap s H}$ and $p_{r H+s H}$ respectively. If $T$ is a closed densely defined linear operator, it has a polar decomposition $T=u|T|$ where $u$ is a partial isometry with $u^{*} u=p_{N(T)^{1}}, u u^{*}=p_{R(T)}$, and $|T|=\left(T^{*} T\right)^{1 / 2}$ is positive selfadjoint. One has $T^{*}=|T| u^{*}$ and $\left|T^{*}\right|=u|T| u^{*}$. If $T \sim \mathfrak{U}$, then $u \in \mathfrak{U}$ and the spectral projections of $|T|$ are in $\mathfrak{A}$. Applying (iii) to $u^{*} u$ we have

$$
\varphi\left(p_{N(T)^{\perp}}\right)=\varphi\left(p_{R(T)}\right) .
$$

If $q_{1}, q_{2} \in \mathfrak{A}$ are projections, letting $T=q_{2}\left(1-q_{1}\right)$ in (0.4) we obtain $\varphi\left(q_{1} \cup q_{2}-q_{1}\right)=$ $\varphi\left(q_{2}-q_{1} \cap q_{2}\right)$, hence

$$
\varphi\left(q_{1} \cup q_{2}\right) \leqq \varphi\left(q_{1}\right)+\varphi\left(q_{2}\right) .
$$

An equality $A=u|A|$ will usually mean that the right-hand side is the polar decomposition of $A$. An equality $A=\int_{0}^{\infty} \lambda d e_{\lambda}$ will usually mean that the right-hand side is the spectral representation of the positive self-adjoint operator $A$. An integral $\int_{a}^{b}$ will mean the integral over the half-open interval $[a, b)$.

A motivating remark. For a positive self-adjoint operator $T \sim \mathfrak{A}$ define the upper integral $\tilde{\varphi}(T)$ by

$$
\tilde{\varphi}(T)=\inf \left\{\sum_{1}^{\infty} \varphi\left(A_{n}\right) \mid A_{n} \in \mathfrak{U}^{+}, \sum_{1}^{\infty} A_{n} \geqq T\right\}
$$


where $\sum_{1}^{\infty} A_{n} \geqq T$ means $\sum_{1}^{\infty}\left(A_{n} x \mid x\right) \geqq(T x \mid x)$ for all $x \in D(T)$. If $\tilde{\varphi}(T)<\infty$, then the domain $D(T)$ is "big" or more precisely $\varphi$-dense in the sense of (1.1). (We show this in Section 5.) In particular, the intersection of two (or even countably many) such domains is still dense in the Hilbert space $H$, and the corresponding operators are necessarily measurable in the sense of Segal. Furthermore, if in (0.6) we change the definition by asking for $\sum A_{n} \geqq T$ on a "big" subspace only, call the result $\bar{\varphi}(T)$ say, then $\bar{\varphi}(T)=$ $\tilde{\varphi}(T)$ (see Section 5) and the formula for $\bar{\varphi}$ is easier to work with than that for $\tilde{\varphi}$. These facts have determined the way in which things are exposed below.

\section{Strongly measurable operators.}

(1.1) Definition. A subspace $S$ of $H$ is called $\varphi$-dense, if for every $\varepsilon>0$ there is a projection $p \in \mathfrak{A}$ with $p H \subset S$ and $\varphi\left(p^{\perp}\right)<\varepsilon$. We say that a property holds almost everywhere (a.e.) on $H$, if it holds on a $\varphi$-dense subspace.

R e mark. If $\mathfrak{Y}$ is $L^{\infty}$ of a finite measure space $(X, B, \mu)$ and $T, T_{n} \sim \mathfrak{A}$ are unbounded operators defined by multiplication with the measurable functions $t, t_{n}$ we have: $T \geqq 0$ a.e. on $H$ if and only if $t \geqq 0$ a.e. on $X$.

If $t_{n} \rightarrow t$ a.e. on $X$, then $T_{n} \rightarrow T$ (strongly) a.e. on $H$. If $T_{n} \rightarrow T$ a.e. on $H$, then $t_{n_{i}} \rightarrow t$ a.e. on $X$ for a suitable subsequence $\left\{t_{n_{i}}\right\}$ (see (3.7)).

(1.2) Remark. The intersection of countably many $\varphi$-dense subspaces is $\varphi$-dense, because $\varphi\left(\left(\cap p_{i}\right)^{\perp}\right) \leqq \sum \varphi\left(p_{i}^{\perp}\right)$ which follows from $\left(\cap p_{i}\right)^{\perp}=\cup p_{i}{ }^{\perp}$ and the inequality $\varphi\left(\cup q_{i}\right) \leqq \sum \varphi\left(q_{i}\right)$. (This last inequality follows from $(0.5)$ by induction and normality.)

(1.3) Definition. Let $N$ be the set of all densely defined closed operators $T$ affiliated to $\mathfrak{A}$. If $T \in N$ has $\varphi$-dense domain, $T$ is called strongly measurable. The set of all strongly measurable operators is denoted by $M$.

For $T \in N$, if $S \subset D(T)$ is a closed subspace, $T p_{S}$ is bounded by the Closed Graph Theorem.

If $A, B \in M$ coincide on a $\varphi$-dense subspace of $H$, one has $A=B$ (see [12], p. 110 for a short proof of this).

(1.4) Lemma. If $B \in M$ and $D$ is a $\varphi$-dense subspace of $H$, the inverse image $B^{-1}(D)$ is $\varphi$-dense.

Proof. Let $r, s \in \mathfrak{U}$ be projections with $r H \subset D, \varphi\left(r^{\perp}\right)<\varepsilon, s H \subset D(B), \varphi\left(s^{\perp}\right)<\varepsilon$. Let $q$ be the projection onto the inverse image of $r H$ under $B s$, that is $q=p_{N\left(r^{\perp} B s\right)}$. By (0.4) we have $\varphi\left(q^{\perp}\right)=\varphi\left(p_{R\left(r^{\perp} B s\right)}\right) \leqq \varphi\left(r^{\perp}\right)<\varepsilon$. Now $(s \cap q) H \subset B^{-1}(D)$ by construction, and $\varphi\left((s \cap q)^{\perp}\right)=$ $\varphi\left(s^{\perp} \cup q^{\perp}\right)<2 \varepsilon$. So $B^{-1}(D)$ is $\varphi$-dense.

(1.5) Proposition. If $A, B \in M$, then $A^{*}, \overline{A+} \bar{B}, \bar{A} \bar{B} \in M$. With these operations (the latter two being called strong sum and strong product) $M$ is a *-algebra.

Proof. a) If $A=u|A| \in M$, we have $|A| \in M$ since $D(|A|)=D(A)$. By (1.4) $A^{*}=|A| u^{*}$ is $\varphi$-densely defined. Being an adjoint, $A^{*}$ is closed.

b) Let $A, B \in M$. Since $A$ and $B$ are $\varphi$-densely defined, so is $A+B$. Since $A^{*}, B^{*} \in M$ (by a)) $A^{*}+B^{*}$ is $\varphi$-densely defined, too. In particular, $(A+B)^{*} \supset A^{*}+B^{*}$ is densely defined, so $A+B$ is closable.

c) For $A, B \in M, A B$ is $\varphi$-densely defined by (1.4). The same applies to $B^{*} A^{*}$, so $(A B)^{*} \supset B^{*} A^{*}$ is densely defined, hence $A B$ is closable. 
d) Using the fact that an operator is affiliated with $\mathfrak{A}$ if and only if its graph is invariant under the operators $U \oplus U$ for unitary $U \in \mathfrak{Q}^{\prime}$, it is routine to check that $A^{*}, \overrightarrow{A+B}, \overrightarrow{A B}$ are affiliated with $\mathfrak{A}$. That $M$ is a *-algebra with these operations, follows from the remark before (1.4). For instance to prove $\overline{\overline{A+B}+C=A+\overline{B+C}}$ it is sufficient to notice that $D(A) \cap D(B) \cap D(C)$ is $\varphi$-dense.

In the sequel we shall often omit closure bars on operators in order to simplify notation.

The section just finished corresponds to part of Section 2 in [14], working with modified definitions. The definition of strong measurability may be considered as implicit in [12], as $M$ is the image of $\tilde{\mathfrak{U}}$ in [12], Theorem 4.

2. Upper integral $\bar{\varphi}$. For $T \in N^{+}$(i.e. $T$ positive self-adjoint and affiliated to $\mathfrak{A}$ ) we define

$$
\bar{\varphi}(T)=\inf \left\{\sum_{1}^{\infty} \varphi\left(A_{n}\right) \mid A_{n} \in \mathfrak{U}^{+}, \sum_{1}^{\infty} A_{n} \geqq T \text { a.e. }\right\} \text {. }
$$

Here $\sum_{1}^{\infty} A_{n} \geqq T$ a.e. means $\sum_{1}^{\infty}\left(A_{n} x \mid x\right) \geqq(T x \mid x)$ for all $x$ in a $\varphi$-dense subspace of $H$, where the infinite sum may take the value $\infty$. Since $\inf \emptyset=\infty$, we have $\bar{\varphi}(T)=\infty$ if $T \notin M$.

(2.1) Proposition. $\bar{\varphi}=\varphi$ on $\mathfrak{A}^{+}$.

Proof. Clearly $\bar{\varphi} \leqq \varphi$ on $\mathfrak{I}^{+}$, so we have to show $\bar{\varphi} \geqq \varphi$. Let $A \in \mathfrak{U}^{+}$and $A_{i} \in \mathfrak{I}^{+}$with $\sum A_{i} \geqq A$ a.e. Then there is a projection $p \in \mathfrak{U}$ with $\sum A_{i} \geqq A$ on $p H$ and $\varphi\left(p^{\lrcorner}\right)<\varepsilon$. By $(0.3)$ we have

$$
\varphi(A)=\varphi(p A p)+\varphi\left(p^{\lrcorner} A p^{\perp}\right) \leqq \varphi(p A p)+\|A\| \varphi\left(p^{\lrcorner}\right)
$$

and by $(0.2)$ since $\sum p A_{i} p \geqq p A p$ :

$$
\begin{aligned}
& \leqq \sum \varphi\left(p A_{i} p\right)+\|A\| \cdot \varepsilon \\
& \leqq \sum \varphi\left(A_{i}\right)+\|A\| \cdot \varepsilon \quad \text { by }(0.1) .
\end{aligned}
$$

So $\varphi(A) \leqq \sum \varphi\left(A_{i}\right)$, hence $\varphi(A) \leqq \bar{\varphi}(A)$.

(2.2) Proposition. For $S, T, T_{n} \in M^{+}, \bar{\varphi}$ satisfies

(i) $S \leqq T$ a.e. implies $\bar{\varphi}(S) \leqq \bar{\varphi}(T)($ Isotony $)$

(ii) $\bar{\varphi}(\lambda T)=\lambda \bar{\varphi}(T)$ for $\lambda \geqq 0$ (Positive homogeneity)

(iii) If $T=\sum_{1}^{\infty} T_{n}$ weakly a.e. (i.e. $(T x \mid y)=\sum_{i}^{\infty}\left(T_{n} x \mid y\right)$ for all $x, y$ in a $\varphi$-dense subspace) then $\bar{\varphi}(T)=\sum_{1}^{\infty} \bar{\varphi}\left(T_{n}\right)$ (Countable additivity).

In particular $\bar{\varphi}$ is positive linear on $\mathrm{M}^{+}$.

Pro of. (i) and (ii) are ciear. We prove (iii) in two steps.

a) First suppose $T=\sum_{1}^{\infty} T_{n}$ weakly a.e. with $T_{n} \in \mathfrak{U}^{+}$. Clearly $\bar{\varphi}(T) \leqq \sum_{1}^{\infty} \varphi\left(T_{n}\right)$. But also $T \geqq \sum_{1}^{k} T_{n}$ a.e., so $\bar{\varphi}(T) \geqq \bar{\varphi}\left(\sum_{1}^{k} T_{n}\right)=\varphi\left(\sum_{1}^{k} T_{n}\right)=\sum_{1}^{k} \varphi\left(T_{n}\right)$. Hence $\bar{\varphi}(T) \geqq \sum_{1}^{\infty} \varphi\left(T_{n}\right)$, so equality holds. 
b) Now let $T=\sum_{1}^{\infty} T_{n}$ weakly a.e., with $T_{n} \in M^{+}$. Choose $T_{n, k} \in \mathfrak{A}^{+}$such that $T_{n}=\sum_{k} T_{n, k}$ weakly a.e. for each $n$. By a) we have $\bar{\varphi}(T)=\sum_{n, k} \varphi\left(T_{n, k}\right)=\sum_{n} \bar{\varphi}\left(T_{n}\right)$.

(2.3) Corollary. For $T=\int_{0}^{\infty} \lambda d e_{\lambda} \in M^{+}$and $p \geqq 1$ we have

$$
\bar{\varphi}\left(T^{p}\right)=\int_{0}^{\infty} \lambda^{p} d \varphi\left(e_{\lambda}\right)
$$

Pro of. Taking $T_{n}=\int_{n}^{n} i^{p} d e_{\lambda}$ we obtain from the last Proposition

$$
\bar{\varphi}\left(T^{p}\right)=\sum \varphi\left(T_{n}\right)=\sum \int_{n}^{n} \lambda^{p} d \varphi\left(e_{\lambda}\right)=\int_{0}^{\infty} \lambda^{p} d \varphi\left(e_{\lambda}\right) .
$$

The following is a noncommutative Beppo Levi Theorem:

(2.4) Theorem. Let $T_{i} \in M^{+}$with $\sum \bar{\varphi}\left(T_{i}\right)<\infty$. There is $T \in M^{+}$such that $\sum T_{i}$ converges weakly a.e. to $T$, and we have $\bar{\varphi}(T)=\sum \bar{\varphi}\left(T_{i}\right)$.

Proof. Let $A_{i k} \in \mathfrak{U}^{+}$with $T_{i}=\sum_{k} A_{i k}$ weakly on $D\left(T_{i}\right)$. Let $D_{0}=\left\{x \in H \mid \sum_{i, k}\left(A_{i k} x \mid x\right)=\right.$ $\left.\sum\left\|A_{i k}^{1 / 2} x\right\|^{2}<\infty\right\}$. Clearly, $D_{0}$ is a linear subspace and $U D_{0} \subset D_{0}$ for unitary $U \in \mathfrak{A}^{\prime}$, so the projection $p$ of $H$ onto $\bar{D}_{0}$ is in $\mathscr{U}$. Since $\sum\left(A_{i k} x \mid x\right)=\infty$ outside $D_{0}$, we have for $n \in \mathbb{N}$

$$
\begin{aligned}
n p^{\perp} & \leqq \sum A_{i k} \\
\text { hence } \quad n \varphi\left(p^{\perp}\right) & \leqq \sum \varphi\left(A_{i k}\right)<\infty,
\end{aligned}
$$

so $\varphi\left(p^{\perp}\right)=0$, that is: $D_{0}$ is dense. The form $\sum A_{i k}$ on $D_{0}$ is closed (easy to check), so there is a self-adjoint positive operator $T$ on $D \subset D_{0}$ with $T=\sum A_{i k}$ weakly on $D$ (see e.g. [13], p. 278). We have $T \sim \mathcal{U}$, so $T \in N$, and assuming for the moment that $D=D(T)$ is $\varphi$-dense, we have by (2.2) $\bar{\varphi}(T)=\sum \varphi\left(A_{i k}\right)=\sum \bar{\varphi}\left(T_{i}\right)$. Clearly, $T=\sum T_{i}$ weakly on the $\varphi$-dense space $\left(\bigcap_{i} D\left(T_{i}\right)\right) \cap D$. It remains to show that $D$ is $\varphi$-dense. If $T=\int \lambda d e_{\lambda}$ and $t>0$, we have $e_{t} H \subset D=D(T)$ and $t e_{t}^{\perp} \leqq T$ on $D$. Since $D$ is a core for the form $\sum A_{i k}$ on $D_{0}$ we have $t e_{t}^{\perp} \leqq \sum A_{i k}$ on $D_{0}$. Outside $D_{0}$ this last inequality holds, too, the form on the right being $\infty$ there. So $t e_{t}^{\perp} \leqq \sum A_{i k}$ a.e. (in fact: everywhere on $H$ ) which implies

$$
t \varphi\left(e_{t}^{\perp}\right) \leqq \sum \varphi\left(A_{i k}\right)<\infty .
$$

Thus we have $\varphi\left(e_{t}^{\perp}\right)<\varepsilon$ for $t$ sufficiently large. Hence $D$ is $\varphi$-dense.

(2.5) Proposition. For $T \in M^{+}$and $B \in \mathfrak{Q}$ we have

$$
\bar{\varphi}\left(B^{*} T B\right) \leqq\|B\|^{2} \ddot{\varphi}(T) .
$$

Proof. Let $A_{i} \in \mathfrak{U}^{+}$with $\sum A_{i} \geqq T$ a.e. By (1.4), we have $\sum B^{*} A_{i} B \geqq B^{*} T B$ a.e., hence $\bar{\varphi}\left(B^{*} T B\right) \leqq \sum \varphi\left(B^{*} A_{i} B\right)=\sum \varphi\left(A_{i}^{1 / 2} B B^{*} A_{i}^{1 / 2}\right) \leqq\|B\|^{2} \sum \varphi\left(A_{i}\right)$. So $\bar{\varphi}\left(B^{*} T B\right) \leqq\|B\|^{2} \bar{\varphi}(T)$.

3. The Banach space $L^{1}$ and the normed sets $L^{p}$. For $1 \leqq p<\infty$ we define $L^{p}=\left\{T \in M \mid \bar{\varphi}\left(|T|^{p}\right)<\infty\right\}$ and $\|T\|_{p}=\bar{\varphi}\left(|T|^{p}\right)^{1 / p}$ for $T \in L^{p}$.

If $|T|=\int \lambda d e_{\lambda}$, then $\|T\|_{p}=\left(\int \lambda^{p} d \varphi\left(e_{\lambda}\right)\right)^{1 / p}$ by $(2.3)$.

(3.1) Proposition. $L^{p}$ is *-invariant and $\left\|T^{*}\right\|_{p}=\|T\|_{p}$. 
Pro of. For $T \in M$ we have $\left|T^{*}\right|^{p}=u|T|^{p} u^{*}$, so by (2.5) $\bar{\varphi}\left(\left|T^{*}\right|^{p}\right) \leqq \bar{\varphi}\left(|T|^{p}\right)$. Replacing $T$ by $T^{*}$, the assertion follows.

Let us note the special case $p=2$ of this proposition:

$$
\bar{\varphi}\left(T T^{*}\right)=\bar{\varphi}\left(T^{*} T\right) \quad \text { for } T \in L^{2} .
$$

To show that $L^{1}$ is a normed linear space and $L^{2}$ is an inner product space, the classical arguments work for unbounded operators, too:

Applying $\bar{\varphi}$ to the inequalities

$$
(A+B)^{*}(A+B) \leqq 2\left(A^{*} A+B^{*} B\right) \text { a.e. for } A, B \in L^{2}
$$

and

$$
(A B)^{*}(A B) \leqq\|A\|^{2} B^{*} B \text { a.e. for } A \in \mathfrak{I}, B \in L^{2}
$$

we see that $L^{2}$ is a left $\mathfrak{U}$-module and

$$
\|A B\|_{2} \leqq\|A\|\|B\|_{2} \text {. }
$$

Since $L^{2}$ is $*$-invariant, it is a two-sided $\mathscr{O}$-module. So $K=\left\{\sum_{1}^{r} A_{i}^{*} B_{i} \mid A_{i}, B_{i} \in L^{2}\right\}$ is a twosided $\mathscr{U}$-module. Since $T=u|T|_{k}^{1 / 2} \cdot|T|^{1 / 2}$ we have $L^{1} \subset K$. Conversely, if $T=\sum_{1}^{k} A_{i}^{*} B_{i} \in K^{*}$, then $T \leqq \sum_{1}^{k}\left(A_{i}^{*} B_{i}+B_{i}^{*} A_{i}\right) \leqq \sum_{1}^{k}\left(A_{i}+B_{i}\right)^{*}\left(A_{i}+B_{i}\right)$ a.e., so $T \in L^{1}$. For arbitrary $T=u|T| \in K$ we have $|T|=u^{*} T \in K^{+} \subset L^{1}$, hence $T \in L^{1}$. So $K=L^{1}$. It follows that $L^{1}$ is linearly generated by $\left(L^{1}\right)^{+}$and we may extend $\bar{\varphi}$ to a linear functional on $L^{1}$ denoted again by $\bar{\varphi}$. By (3.2) and polarization we obtain $\bar{\varphi}\left(S T^{*}\right)=\bar{\varphi}\left(T^{*} S\right)$, hence

$$
\bar{\varphi}(S T)=\bar{\varphi}(T S) \quad \text { for } S, T \in L^{2} \text {. }
$$

Using $T=u|T|^{1 / 2}|T|^{1 / 2}$ and applying (3.4) twice we see that (3.4) also holds for $S \in \mathfrak{A}, T \in L^{1}$. We have $\bar{\varphi}\left(T^{*}\right)=\overline{\bar{\varphi}(T)}$ since $\bar{\varphi}$ is positive on $\left(L^{1}\right)^{+}$. So $(S \mid T)=\bar{\varphi}\left(T^{*} S\right)$ is an inner product on $L^{2}$. For $S \in \mathcal{Q} I$ and $T=u|T| \in L^{1}$ we have by the Cauchy-Schwarz inequality

$$
\begin{aligned}
|\bar{\varphi}(S T)| & =\left|\bar{\varphi}\left(S u|T|^{1 / 2}|T|^{1 / 2}\right)\right| \\
& \leqq\left\|S u|T|^{1 / 2}\right\|_{2}\left\|\left._{T} T\right|^{1 / 2}\right\|_{2} \\
& \leqq\|S u\|\left\|\left.T\right|^{1 / 2}\right\|_{2}^{2} \\
& \leqq\|S\| \bar{\varphi}(|T|) .
\end{aligned}
$$

This together with $\bar{\varphi}\left(u^{*} T\right)=\bar{\varphi}(|T|)$ shows that

$$
\|T\|_{1}=\sup \{\bar{\varphi}(S T) \mid S \in \mathfrak{U},\|S\| \leqq 1\} .
$$

So the map $T \mapsto \vec{\varphi}(\cdot T)$ is linear isometric from $L^{\mathfrak{1}}$ to the dual of $\mathfrak{A}$, in particular \|\|$_{1}$ satisfies the triangle inequality. If $\|T\|_{1}=0$ and $|T|=\int \lambda d e_{\lambda}$, then $0=\int_{0}^{\infty} \lambda d \varphi\left(e_{\lambda}\right) \geqq \int_{0}^{n} \lambda d \varphi\left(e_{\lambda}\right)=$ $\varphi\left(\int_{0}^{n} \lambda d e_{\lambda}\right)$, so $\int_{0}^{n} \lambda d e_{\lambda}=0$ since $\varphi$ is faithful, hence $|T|=0$, i.e. $T=0$. Thus $L^{l}$ is a normed linear space.

$\mathrm{R} \mathrm{e} \mathrm{m} \mathrm{a} \mathrm{r} \mathrm{k.} \mathrm{Another} \mathrm{proof} \mathrm{that} L^{1}$ is a normed linear space can be obtained as follows:

(i) For $A \in M, A=v|A|$, one has $4 \operatorname{Re} A \leqq(1+v)|A|(1+v)^{*}$ a.e., which follows from $0 \leqq(1-v)|A|(1-v)^{*}$ by adding $4 \operatorname{Re} A=2\left(A+A^{*}\right)=2\left(v|A|+|A| v^{*}\right)$ on both sides.

(ii) For $C \in \mathscr{U}, B \in M$ one has $\|C B\|_{1} \leqq\|C\|\|B\|_{1}$. This follows from $B^{*} C^{*} C B \leqq\|C\|^{2} B^{*} B$ a.e. and the fact that the square root is operator a.e.-monotone, so $|C B| \leqq \| C|||B|$ a.e.

(iii) For $S, T \in L^{1}$ one has $S+T=u|S+T|$, hence

$$
\begin{aligned}
|S+T| & =u^{*} S+u^{*} T=\operatorname{Re} u^{*} S+\operatorname{Re} u^{*} T \\
& \leqq \frac{1}{4}(1+v)\left|u^{*} S\right|(1+v)^{*}+\frac{1}{4}(1+w)\left|u^{*} T\right|(1+w)^{*} \quad \text { a.e. } \quad \text { by (i). }
\end{aligned}
$$


Applying $\bar{\varphi}$ and using (2.5) we obtain

$$
\|S+T\|_{1} \leqq\left\|u^{*} S\right\|_{1}+\left\|u^{*} T\right\|_{1} \leqq\|S\|_{1}+\|T\|_{1} \quad \text { by (ii). }
$$

This remark has been inspired by [1], Section 2 .

(3.5) Theorem. $L^{1}$ is complete.

Proof. It suffices to show that $\sum\left\|T_{n}\right\|_{1}<\infty$ implies convergence of $\sum T_{n}$ in $L^{1}$. Decomposing into positive parts and applying Theorem (2.4) we obtain the desired result.

For completeness we include the main convergence theorems. Readers who want to get to $L^{p}$ quickly, should skip to 3.9 or Section 4.

(3.6) Monotone Convergence Theorem. Let $\left\{T_{n}\right\}$ be a sequence in $L^{1}$ with $T_{n+1} \geqq T_{n}$ on $\bigcap_{1}^{\infty} D\left(T_{k}\right)$ and suppose $\sup _{n} \bar{\varphi}\left(T_{n}\right)<\infty$. Then there is $T \in L^{1}$ such that $T_{n} \rightarrow T$ weakly a.e. and $T_{n} \rightarrow T$ in $L^{1}$.

Proof. We have $S_{n}=T_{n+1}-T_{n} \geqq 0$ a.e. and this readily implies $S_{n} \in\left(L^{1}\right)^{+}$. Applying Theorem (2.4) to $\left\{S_{i}\right\}$ we obtain that $T_{n}=T_{1}+\sum_{1} S_{i}$ converges weakly a.e. to some $T=T_{1}+S$, and $\left\|T-T_{n}\right\|_{1}=\| \sum_{n}^{\infty} S_{i} \mid=\sum_{n}^{\infty} \bar{\varphi}\left(S_{i}\right) \rightarrow 0$ for $n \rightarrow \infty$.

The following theorem can probably be reduced to Theorem 4.13, p. 85 in [17], but for the reader's convenience we include a direct proof.

(3.7) Egoroff's Theorem. Let $T, T_{n} \in M$ with $T_{n} \rightarrow T$ strongly a.e. and let $q \in \mathfrak{N}$ be a projection with $\varphi(q)<\infty$. Then there is a subsequence $\left\{T_{n_{i}}\right\}$ which converges to $T$ "almost uniformly on $q H$ ", i.e. for every $\varepsilon>0$ there is a projection $p \in \mathfrak{U}$ with $p \leqq q$, $\varphi(q-p)<\varepsilon$ and such that $T_{\boldsymbol{n}_{\boldsymbol{i}}} \rightarrow T$ in the uniform norm on $p H$.

Proof. a) Let $S_{n}=T-T_{n}$. We first show that, given $n_{0} \in \mathbb{N}$ and $t>0$, there is $n^{\prime}>n_{0}$ and a projection $p \in \mathfrak{U}$ such that $p \leqq q, \varphi(q-p)<t$ and the uniform norm of $S_{n^{\prime}}$ on $p H$ is less than $t$.

(i) Since $D=\left\{x \in H \mid S_{n} x \rightarrow 0\right\}$ is $\varphi$-dense by assumption, there is a projection $r \in \mathfrak{U}$ with $r H \subset D, \varphi\left(r^{\perp}\right)<\eta$. The projection $q^{\prime}=q \cap r$ satisfies $q^{\prime} H \subset D, q^{\prime} \leqq q$ and $\varphi\left(q-q^{\prime}\right) \leqq \varphi\left(r^{\perp}\right)<\eta$ (since $q-q \cap r \sim q \cup r-r \leqq r^{\perp}$ ). So, replacing $q$ by $q^{\prime}$, we may assume $q H \subset D$. Looking at the (actually bounded) operators $S_{n} q$ and replacing them by their absolute value, we may assume that $S_{n}$ is positive and maps $q H$ into itself, that $q H=H$ and therefore $\varphi$ is finite.

(ii) If $S_{n}=\int \hat{\lambda} d e_{\lambda, n}$ and $t>0$ consider the projections $e_{t, n}$. Since $S_{n} x \rightarrow 0$ we have $e_{t, n} x \rightarrow x$ for $x \in H$. This implies $\varphi\left(e_{t, n}\right) \rightarrow \varphi(1)$ (as the convergence need not be monotone, one uses $\varphi(A)=\sum_{i}\left(A x_{i} \mid x_{i}\right)$ for this). Since $\varphi$ is finite, it follows $\varphi\left(e_{t, n}^{\perp}\right) \rightarrow 0$ for $n \rightarrow \infty$. So there is $n^{\prime}>n_{0}$ with $\varphi\left(e_{t, n^{\prime}}^{\perp}\right)<t$. Now $n^{\prime}$ and $p=e_{t, n^{\prime}}$ are as required.

b) Let $t_{i}>0$ with $\sum_{1}^{\infty} t_{i}<\infty$. Choose an increasing sequence $n_{i}$ and projections $p_{i}$ such that $n_{i}$, $p_{i}$ are as in a) for $t=t_{i}$ and $n_{0}=n_{i-1}$. We assert that $T_{n_{i}} \rightarrow T$ almost uniformly on $q H$. Let $\varepsilon>0$. There is $k \in \mathbb{N}$ such that $\sum_{k}^{\infty} t_{i}<\varepsilon$. Let $p=\bigcap_{k}^{\infty} p_{i}$. We have $p \leqq q, \varphi(q-p) \leqq \sum_{k}^{\infty} t_{i}<\varepsilon$. For $i \geqq k$ we have $p \leqq p_{i}$, hence $\left\|S_{n_{i}} x\right\| \leqq t_{\mathrm{i}}\|x\|$ for $x \in p H$. As $t_{i} \rightarrow 0$ for $i \rightarrow \infty$ the theorem is proved.

One might think that in the above theorem the conclusion should hold for the full sequence $\left\{T_{n}\right\}$ (like in the classical Egoroff Theorem for functions), but this is erroneous. Even in the commutative case the conclusion does not hold for the full sequence. We see this from the following 
Ex a mple. Let $H=L^{2}(0,1), \mathfrak{U}=L^{\bullet}(0,1)$ (we identify functions with the multiplication operators defined by them) and $\varphi(f)=\int_{0}^{1} f(x) d x$ for $f \in \mathfrak{U}^{+}$. Let $t_{\boldsymbol{n}} \in \mathfrak{U}$ be the characteristic functions of the intervals $\left(0, \frac{1}{2}\right),\left(\frac{1}{2}, 1\right),\left(0, \frac{1}{3}\right),\left(\frac{1}{3}, \frac{2}{3}\right),\left(\frac{2}{3}, 1\right),\left(0, \frac{1}{4}\right) \ldots$ For $g \in L^{2}(0,1)$ we have $\| t_{n} g:{ }_{2} \rightarrow 0$, so $t_{n} \rightarrow 0$ strongly (everywhere) on $L^{2}(0,1)$. Clearly $t_{n}$ does not converge almost uniformly on $L^{2}(0,1)$ to 0 (we even have: $t_{n} \rightarrow 0$ almost uniformly on $p H$ implies $p=0$ ).

(3.8) Dominated Convergence Theorem. Let $T, T_{n} \in M$ with $T_{n} \rightarrow T$ strongly a.e. and suppose there is $A \in\left(L^{1}\right)^{+}$with $\left|T-T_{n}\right| \leqq A$ a.e. Then $T_{n} \rightarrow T$ in $L^{1}$.

P roof. It suffices to prove the assertion for a subsequence of $\left\{T_{n}\right\}$. Let $\left\{T_{n_{i}}\right\}$ be as in Egoroff's Theorem. Let $\varepsilon>0$ and $A=\int \lambda d e_{\lambda}$. Choose $t>s>0$ such that the projection $e=e_{t}-e_{s}$ satisfies $\bar{\varphi}\left(A e^{\perp}\right)<\varepsilon$. For $\varepsilon^{\prime}=\frac{\varepsilon}{t}$ and $q=e$ let $p$ be as in Egoroff's Theorem. Since $1=p+(e-p)+e^{\perp}$ we have

$$
\bar{\varphi}\left(\left|T-T_{n_{i}}\right|\right)=\bar{\varphi}\left(\left|T-T_{n_{i}}\right| p\right)+\bar{\varphi}\left(\left|T-T_{n_{\mathrm{i}}}\right|(e-p)\right)+\bar{\varphi}\left(\left|T-T_{n_{\mathrm{i}}}\right| e^{\perp}\right) .
$$

The first term on the right-hand side equals $\bar{\varphi}\left(p\left|T-T_{n_{i}}\right| p\right)$ and so can be estimated by $\varphi(p)$ times the uniform norm of $T-T_{n_{i}}$ on $p H$, hence is $<\varepsilon$ for $i>i_{0}$. The second term can be estimated by $\bar{\varphi}(A(e-p))$, hence is $\leqq\|A e\| \varphi(e-p) \leqq t \cdot \frac{\varepsilon}{t}=\varepsilon$. The third term is less than $\bar{\varphi}\left(A e^{\perp}\right)<\varepsilon$. So $\| T-T_{n_{\mathrm{i}}} \mathrm{l}_{1}<3 \varepsilon$ for $i>i_{0}$, which proves the theorem.

The reader will have noticed that the proof comes from standard measure theory.

By standard arguments again we obtain

(3.9) Theorem. The map $B \mapsto \bar{\varphi}(\cdot B)$ is an isometric isomorphism from $L^{1}$ onto the predual $\mathfrak{A}_{*}$ of $\mathfrak{A}$, the space of all ultraweakly continuous linear functionals on $\mathfrak{A}$.

Proof. As we have already shown before (3.5), the map $B \mapsto f_{B} \stackrel{\text { def }}{=} \bar{\varphi}(\cdot B)$ is an isometric isomorphism from $L^{1}$ onto a linear subspace $S$ of the dual $\mathfrak{U}^{*}$. Since $L^{1}$ is complete, $S$ is closed. To show that the functionals $f_{B}$ are ultraweakly continuous it suffices to consider $B \in L^{1} \cap \mathfrak{Q}^{+}$(in which case $f_{B}$ is positive, since $\bar{\varphi}(A B)=\varphi\left(B^{1 / 2} A B^{1 / 2}\right) \geqq 0$ for $\left.A \in \mathfrak{I}^{+}\right)$and to prove normality for $f_{B}$. If $A_{\mu}>A$ in $\mathfrak{A}^{+}$weakly then $B^{1 / 2} A_{\mu} B^{1 / 2}>B^{1 / 2} A B^{1 / 2}$ weakly, so $\varphi\left(A_{\mu} B\right)>\varphi(A B)$, hence $f_{B}$ is normal. This shows $S \subset \mathfrak{U}_{*}$. To obtain $S=\mathfrak{U}_{*}$, by Hahn-Banach it suffices to find for a given $0 \neq A \in \mathfrak{Q}$ some $B \in L^{1}$ such that $f_{B}(A) \neq 0$. Let $p \in \mathfrak{U}$ be a projection with $\lambda p \leqq|A|$ for some $\lambda>0$ and $0<\varphi(p)<\infty$ and take $B=p u^{*}$ (where $A=u|A|$ ). Then $\bar{\varphi}(B A)=$ $\bar{\varphi}(p|A|)=\varphi(p|A| p) \geqq \lambda \varphi(p)>0$. This proves the theorem.

Let us note that Theorem (3.9) also is a Radon-Nikodym result. The isomorphism $f_{B} \mapsto B$ from $\mathfrak{U}_{*}$ onto $L^{1}$ assigns to each functional in $\mathfrak{U}_{*}$ its Radon-Nikodym derivative with respect to $\bar{\varphi}$.

4. Identification of $\boldsymbol{L}^{p}$ as interpolation space. For the reader's convenience we mention some facts from complex interpolation theory.

Let the Banach spaces $A_{0}$ and $A_{1}$ be compatible, i.e. there is a Hausdorff topological linear space $X$ in which $A_{0}$ and $A_{1}$ are continuously embedded. The algebraic sum $\sum=\sum\left(A_{0}, A_{1}\right)=$ $A_{0}+A_{1}$ is a Banach space with the norm

$$
\|a\|_{\Sigma}=\inf \left\{\left\|a_{0}\right\|_{A_{\bullet}}+\left\|a_{1}\right\|_{A_{1}} \mid a_{0} \in A_{0}, a_{1} \in A_{1}, a=a_{0}+a_{1}\right\} .
$$

The inclusion of $\sum$ into $X$ is continuous. Let $F=F\left(A_{0}, A_{1}\right)$ be the space of all functions $f$ with values in $\sum$ which for \|\|$_{\Sigma}$ are continuous and bounded on the strip $S=\{z \in \mathbb{C} \mid 0 \leqq \operatorname{Re} z \leqq 1\}$ and holomorphic in the interior $0<\operatorname{Re} z<1$, and such that the functions $t \mapsto f(k+i t), k=0,1$ 
are continuous and bounded from $\mathbb{R}$ to $A_{k}$ (some authors include the condition that these boundary functions vanish at infinity. The interpolation spaces to be constructed are identical in both cases). $F$ is a Banach space when equipped with the norm

$$
\|f\|=\max \left\{\sup _{t \in \mathbb{R}}\|f(i t)\|_{A 0}, \quad \sup _{t \in \mathbb{R}}\|f(1+i t)\|_{A_{1}}\right\} .
$$

For $0<\theta<1$, the complex interpolation space $\left(A_{0}, A_{1}\right)_{\theta}$ is defined to be the space $\left\{a \in \sum \mid a=\right.$ $f(\theta)$ for some $f \in F\}$ with the norm $\|a\|_{(\theta)}=\inf \{\|f\|\{a=f(\theta), f \in F\}$. It is a Banach space and $\|a\|_{(\theta)} \geqq\|a\|_{\Sigma}$, so the inclusion into $\sum$ and hence the inclusion into $X$ are continuous.

We want to apply this to $A_{0}=\mathfrak{U}$ and $A_{1}=L^{1}$. The space $X$ containing them is $M$ with the topology of convergence in measure, i.e. the topology having the following sets $N(\varepsilon)$ as a neighbourhood basis at $0: N(\varepsilon)=\{T \in M \mid$ there is a projection $p \in \mathfrak{U}$ with $p H \subset D(T),\|T p\|<\varepsilon$, and $\left.\varphi\left(p^{\perp}\right)<\varepsilon\right\}$.

The inclusion $\mathfrak{A} \subset M$ is clearly continuous. So is the inclusion $L^{p} \subset M$ for every $p \geqq 1^{*}$ ): If $\|T\|_{p}^{p}<\varepsilon^{1+p}$ and $|T|=\int \lambda d e_{\lambda}$, we have $\left\|T e_{\varepsilon}\right\| \leqq \varepsilon$ and $\varepsilon^{p} e_{\varepsilon}^{\perp} \leqq|T|^{p}$, so $\varepsilon^{p} \varphi\left(e_{\varepsilon}^{\perp}\right) \leqq$ $\bar{\varphi}\left(|T|^{p}\right)<\varepsilon^{1+p}$, hence $\varphi\left(e_{\varepsilon}^{\perp}\right)<\varepsilon$. So $T \in N(\varepsilon)$.

The space $M$ is Hausdorff: if $T \in N(\varepsilon)$ for all $\varepsilon>0$ one has $T=0$ a.e., hence $T=0$.

Let us now identify $L^{p}$ as $\left(\mathfrak{A}, L^{1}\right)_{\theta}$ where $\theta=\frac{1}{p}$. The proof of the following theorem is almost the classical one for functions (see [2]).

(4.1) Theorem. $L^{p}=\left(\mathfrak{N}, L^{1}\right)_{\frac{1}{p}}$ with equal norms. In particular, $L^{p}$ is a linear space, \|\|$_{p}$ is a norm, and $L^{p}$ is complete under \|\|$_{p}$.

Proof. a) Let $A=u|A| \in L^{p}$ and suppose first that $|A|$ has a spectral representation $\int_{a}^{b} \lambda d e_{\lambda}$. with $0<a<b<\infty$. Then the function $f: z \mapsto \rightarrow u|A|^{p z}$ is in $F\left(\mathfrak{H}, L^{1}\right)$ (this is no problem since all norms are calculated on the space $[a, b)$ with the measurs, $\left.d \varphi\left(e_{\lambda}\right)\right)$. If we assume $\|A\|_{p}=1$, we obtain $\|f\|=1$. Then $A=f\left(\frac{1}{p}\right) \in\left(\mathfrak{M}, L^{1}\right)_{\frac{1}{p}}$ and $\|A\|_{\left(\frac{1}{p}\right)} \leqq 1=\|A\|_{p}$.

If $A=u|A| \in L^{p}$ is arbitrary, we can "cut" $A$ into pieces $A_{n}$ of the above form such that $A-\sum_{1}^{k} A_{n} \|_{p} \rightarrow 0$ and $\sum_{1}^{\infty}\left\|A_{n}\right\|_{p}<\infty$. By the above, the series $\sum A_{n}$ converges absolutely in $\left(\Re, L^{1}\right)_{\frac{1}{p}}$. To see that the limit is the same as in $L^{p}$, namely $A$, it suffices to recall that convergence in $L^{p}$ as well as convergence in $\left(\mathfrak{A}, L^{1}\right)_{1}$ implies convergence in $M$.

b) Let $q$ be the conjugate index of ${ }^{p} p$. Let $A \in\left(\mathfrak{U}, L^{1}\right)_{\frac{1}{p}} \subset \sum\left(\mathfrak{U}, L^{1}\right)$. Then in particular $A \in M$. If $A=u|A|$, then because of the fact that every nonzero projection $r \in \mathfrak{Q}$ dominates a projection $s \in \mathfrak{A}$ with $0<\varphi(s)<\infty$, the norm $\|A\|_{p}$ (be it finite or infinite) can be obtained as the limit of expressions $\bar{\varphi}(B|A|)$ where $B$ has $\|B\|_{q}=1$ and is of the form $\sum_{i}^{k} a_{i} p_{i}$ with $a_{i}>0$ and $p_{i}$ pairwise orthogonal projections in $2 \mathfrak{l}$ commuting with $|A|$ and such that $\varphi\left(p_{i}\right)<\infty$. If $B$ is such, let $g(z)=B^{q\{1-z)} u^{*}=\left(\sum_{1}^{k} a_{i}^{q(1-z)} p_{i}\right) u^{*}$ which is bounded and continuous from the strip

$\left.{ }^{*}\right)$ For $p>1$, at this point we don't know yet that $L^{p}$ is a linear space. So by "continuous inclusion" we mean: If $T, T_{n} \in L^{p}$ and $\left\|T-T_{n}\right\|_{p} \rightarrow 0$, then $T-T_{n} \rightarrow 0$ in measure. 
$0 \leqq \operatorname{Re} z \leqq 1$ to $\left(L^{1} \cap \mathfrak{A},\|\|_{1}+\|\|\right)$ and holomorphic in the interior $0<\operatorname{Re} z<1$. Let now $f \in F\left(\mathfrak{A}, L^{1}\right)$ with $f\left(\frac{1}{p}\right)=A$ and $\|f\|<\|A\|_{\left(\frac{1}{p}\right)}+\varepsilon$. The function

$$
h(z)=\langle g(z), f(z)\rangle=\bar{\varphi}(g(z) f(z))
$$

is bounded and continuous on the strip and holomorphic in the interier. On the line $\operatorname{Re} z=0$ we have $|h(z)| \leqq\|g(z)\|_{1}\|f(z)\| \leqq\left\|B^{g(1-z)}\right\|_{1}\|f\|=\|f\|$, on the line $\operatorname{Re} z=1$ we have $|h(z)| \leqq\|g(z)\|\|f(z)\|_{1} \leqq 1 \cdot\|f\|$. So by the three lines theorem we obtain for $z=\frac{1}{p}$

$$
\left|h\left(\frac{1}{p}\right)\right|=\bar{\varphi}(B|A|) \leqq\|f\| \leqq\|A\|_{\left(\frac{1}{p}\right)}+\varepsilon .
$$

Hence $\|A\|_{p} \leqq\|A\|_{\left(\frac{1}{p}\right)}$ which concludes the proof.

R e m a r k. Michael Cowling pointed out to me that the complex method of interpolation can be modified, by allowing the functions $f(z)$ to be weakly continuous only in $S$, and norm continuous on just one boundary line. The interpolation space which arises in this way is the same as in the classical construction. Using this modified interpolation method, which is described in detail in [16], one can avoid the "cutting into pieces" in a) of the above proof.

The rest is an easy consequence of well-known facts from complex interpolation:

(4.2) Corollary. (i) $L^{2}$ is a Hilberi space.

(ii) $L^{p}$ is reflexive for $1<p<\infty$.

(iii) $\left(L^{p}\right)^{*}=L^{q}$ where $\frac{1}{p}+\frac{1}{q}=1$.

Proof. (i) Being an interpolation space, $L^{2}$ is a Banach space, and we have already seen that its norm comes from an inner product.

(ii) This is obtained by interpolation between $L^{1}$ and $L^{2}$, and between $L^{2}$ and $\mathfrak{A}$, using that $L^{2}$ is reflexive (see [3], 2.12).

(iii) Is true for $1 \leqq p \leqq 2$ by interpolation since $\left(L^{1}\right)^{*}=\mathfrak{A} \stackrel{\text { def }}{=} L^{\infty}$ and $\left(L^{2}\right)^{*}=L^{2}$ (see [2], Corollary 4.5.2). Since $L^{p}$ is reflexive by (ii), the result holds for $2 \leqq p<\infty$, too.

\section{Remarks.}

a) Alternative description of the upper integral and a stronger variant of our approach.

For $T \in N^{+}$let $\tilde{\varphi}(T)=\inf \left\{\sum_{1}^{\infty} \varphi\left(A_{n}\right) \mid A_{n} \in \mathfrak{U}^{+}, \sum_{1}^{\infty} A_{n} \geqq T\right.$ on $\left.D(T)\right\}$.

We want to show that $\check{\varphi}(T)<\infty$ implies that $D(T)$ is $\varphi$-dense, and that $\tilde{\varphi}=\vec{\varphi}$ on $N^{+}$. This is done in several steps, (5.1) to (5.5).

$$
\tilde{\varphi}=\varphi \quad \text { on } \quad \mathfrak{U}^{+}
$$

Proof. Clearly $\check{\varphi} \leqq \varphi$ on $\mathfrak{A}^{+}$. The reverse inequality follows from

$$
A, A_{i} \in \mathfrak{U}^{+}, \sum_{1}^{\infty} A_{i} \geqq A \Rightarrow \sum_{1}^{\infty} \varphi\left(A_{i}\right) \geqq \varphi(A)
$$

which is a special case of $(0.2)$. 
(5.3) Proposition. Let $T \in N^{+}, A_{i} \in \mathfrak{Q}^{+}$for natural $i$, and let $\left\{p_{n}\right\}$ be a sequence of projections in U with $\sum p_{n}=1$ weakly, $p_{n} H \subset D(T)$, and $\sum A_{i} \geqq T$ on $p_{n} H$ for every $n$. Then $\tilde{\varphi}(T) \leqq \sum \varphi\left(A_{i}\right)$.

Proof. If $T=\int_{0}^{\infty} \lambda d e_{\lambda}$, let $T_{m}=\int_{m}^{m} \lambda d e_{\lambda}$. Since $T=\sum T_{m}$ on $D(T)$, we have $\tilde{\varphi}(T) \leqq \sum \varphi\left(T_{m}\right)$. It thus suffices to show $\sum_{1}^{k} \varphi\left(T_{m}\right) \leqq \sum_{1}^{\infty} \varphi\left(A_{i}\right)$ for every $k$. Let $A=\sum_{1}^{k} T_{m}$. Since $A \leqq T \leqq \sum A_{i}$ on $p_{n} H$ for every $n$, we obtain using (0.3) and (5.2) $\varphi(A)=\sum_{n} \varphi\left(p_{n} A p_{n}\right) \leqq \sum_{n} \sum_{i} \varphi\left(p_{n} A_{i} p_{n}\right)=\sum_{i} \varphi\left(A_{i}\right)$.

(5.4) Proposition. Let $T \in N^{+}$with $\tilde{\varphi}(T)<\infty$. Then $D(T)$ is $\varphi$-dense.

Proof. Let $T=\int_{0}^{\infty} \lambda d e_{\lambda}$ and let $\varepsilon>0$. Let $A_{i} \in \mathfrak{U}^{+}$with $T \leqq \sum A_{i}$ on $D(T)$ and $\sum \varphi\left(A_{i}\right)<\tilde{\varphi}(T)+1$. For $t>0$ we have $t e_{\mathrm{t}}{ }^{1} \leqq T \leqq \sum A_{i}$ on $D(T)$. Define projections $p_{n}$ by $p_{1}=e_{1}, p_{n}=e_{n}-e_{n-1}$ for $n>1$. Applying (5.3) with $t \cdot e_{t}^{\perp}$ in place of $T$ there, we obtain $\varphi\left(t \cdot e_{t}^{\perp}\right)=\tilde{\varphi}\left(t \cdot e_{t}^{\perp}\right) \leqq \sum \varphi\left(A_{i}\right)<\tilde{\varphi}(T)+1$. Hence $\varphi\left(e_{t}^{\perp}\right) \leqq \frac{\tilde{\varphi}(T)+1}{t}<\varepsilon$ for $t$ large enough. Since $e_{t} H \subset D(T)$, this shows that $D(T)$ is $\varphi$-dense.

$$
\tilde{\varphi}(T)=\bar{\varphi}(T) \quad \text { for } T \in N^{+} .
$$

Proof. (i) Suppose $\tilde{\varphi}(T)<\infty$. By (5.4) an inequality $\sum A_{n} \geqq T$ on $D(T)$ is an inequality on a $\varphi$-dense space, so $\bar{\varphi}(T) \leqq \tilde{\varphi}(T)$.

(ii) Suppose $\bar{\varphi}(T)<\infty, T=\int_{0}^{\infty} \lambda d e_{\hat{\lambda}}$. Let $A_{i} \in \mathfrak{N}^{+}$with $T \leqq \sum A_{i}$ on a $\varphi$-dense subspace $D \subset D(T)$ of $H$. There are projections $p_{n} \in \mathscr{U}$ with $p_{n} H \subset D$ and $\sum p_{n}=1$ weakly. By (5.3) we have $\tilde{\varphi}(T) \leqq \sum \varphi\left(A_{i}\right)$. Hence $\tilde{\varphi}(T) \leqq \bar{\varphi}(T)$.

There is another description of $\bar{\varphi}$ :

Call a subspace $D$ of $H \mathscr{U}$-dense, if there is a sequence of projections $p_{n} \in \mathfrak{A}$ with $p_{n} H \subset D$ and $\sum p_{n}=1$ weakly. For example, the domain $D(T)$ of any $T \in N$ is $\mathscr{U}$-dense. (Use spectral projections of $|T|$ for $p_{n}$.) If for $T \in N^{+}$we define

$$
\hat{\varphi}(T)=\inf \left\{\sum_{1}^{\infty} \varphi\left(A_{n}\right) \mid A_{n} \in \mathfrak{A}^{+}, \sum_{1}^{\infty} A_{n} \geqq T \text { on an } \mathfrak{A} \text {-dense subspace of } D(T)\right\},
$$

then the proof of (5.5) shows that $\tilde{\varphi}(T)=\hat{\varphi}(T)$.

Despite the equality $\bar{\varphi}=\tilde{\varphi}=\hat{\varphi}$ on $N^{+}$, there is an essential difference: $\bar{\varphi}(T)<\infty$ presupposes that $T$ is defined a.e. (i.e. it presupposes that $T \in M$ ) whereas this fact is a consequence of $\tilde{\varphi}(T)<\infty$ or $\hat{\varphi}(T)<\infty$ (as just seen above). Because of this, our approach to integration with $\tilde{\varphi}$ or $\hat{\varphi}$ instead of $\bar{\varphi}$ would be stronger (in many places above it suffices to assume $T \in N$ rather than $T \in M$ ). On the other hand such an approach would cause more work in Section 2.

\section{b) Convergence of measurable operators.}

If $\left\{T_{n}\right\}$ is a sequence in $M$ which is Cauchy in measure, there is a subsequence $\left\{T_{n i}\right\}$ converging almost uniformly on $H$ to some $T \in M$ (after a reduction to the self-adjoint case the proof is roughly the same as for functions). Since almost uniform convergence on $H$ implies convergence in measure and strong convergence a.e. we have (like in the classical case of functions):

(i) $M$ with the topology of convergence in measure is complete.

(ii) If $T_{n} \rightarrow T$ in measure, there is a subsequence $\left\{T_{n k}\right\}$ with $T_{n k} \rightarrow T$ strongly a.e.

If $\varphi$ is finite, in (ii) we may exchange "in measure" and "strongly a.e." by each other because of Egoroff's Theorem. If $\varphi$ is infinite, like in the classical case of functions one easily constructs examples with $T_{n} \rightarrow T$ strongly a.e. but such that no subsequence of $\left\{T_{n}\right\}$ converges to $T$ in measure. 


\section{References}

[1] C. A. Akemann, J. Anderson and G. K. Pedersen, Triangle inequalities in operator algebras. Linear and Mulitlinear Algebra 11, 167-178 (1982).

[2] J. BERGH and J. LÖFSTRÖM, Interpolation spaces. Berlin-Heidelberg-New York 1976.

[3] A. P. CALDERón, Intermediate spaces and interpolation, the complex method. Studia Math. 24, 113-190 (1964).

[4] J. DiXmier, Formes linéaires sur un anneau d'opérateurs. Bull. Soc. Math. France 81, 9-39 (1953).

[5] J. DiXmIER, Les algèbres d'opérateurs dans l'espace Hilbertien. Paris 1969.

[6] P.R. Halmos, Measure Theory. New York-Cincinnati-Toronto-London-Melbourne 1950.

[7] T. Kato, Perturbation Theory for Linear Operators. Berlin-Heidelberg-New York 1976.

[8] R. A. KUNZE, $L^{p}$ Fourier transforms on locally compact unimodular groups. Trans. Amer. Math. Soc. 89, 519-540 (1958).

[9] M. LEINERT, Daniell-Stone integration without the lattice condition. Arch. Math. 38, 258-265 (1982).

[10] M. LeINERT, On integration with respect to a trace. In: Aspects of positivity in Functional Analysis, (ed. Nagel et al.) North Holland Math. Stud. 122, $231-239$ (1986).

[11] M. LEINERT, Integration with respect to a weight. Internat. J. Math. 2, 177-182 (1991).

[12] E. NeLSON, Notes on non-commutative integration. J. Funct. Anal. 15, 103-116 (1974).

[13] M. ReED and B. Simon, Methods of Modern Mathematical Physics I. New York-London 1972.

[14] I. Segal, A non-commutative extension of abstract integration. Ann. of Math. (2) 57, $401-457$ (1953).

[15] W. F. STINESPRING, Integration theorems for gages and duality for unimodular groups. Trans. Amer. Math. Soc. 90, 15-56 (1959).

[16] L. SERvideI, Different methods of complex interpolation. Bull. Austral. Math. Soc. 40, 389-395 (1989).

[17] M. TAKESAKI, Theory of Operator Algebras I. Berlin-Heidelberg-New York 1979.

Eingegangen am 25.1.1991*)

Anschrift des Autors:

Michael Leinert

Institut für Angewandte Mathematik

Universität Heidelberg

DW-6900 Heidelberg

*) Eine leicht modifizierte Fassung ging am 10.6.1992 ein. 